University of South Carolina

Scholar Commons

$4-15-2005$

\title{
Long-Lived Spin Coherence States in Semiconductor Heterostructures
}

Yuriy V. Pershin Dr

University of South Carolina - Columbia, pershin@physics.sc.edu

Follow this and additional works at: https://scholarcommons.sc.edu/phys_facpub

Part of the Physics Commons

\section{Publication Info}

Published in Physical Review B, ed. Gene D. Sprouse, Volume 71, Issue 15, 2005, pages

155317-1-155317-5.

Pershin, Y. V. (2005). Long-lived spin coherence states in semiconductor heterostructures. Physical

Review B, 71(15), 155317-1 - 155317-5. DOI; 10.1103/PhysRevB.71.155317

(C) Physical Review B, 2005, American Physical Society

This Article is brought to you by the Physics and Astronomy, Department of at Scholar Commons. It has been accepted for inclusion in Faculty Publications by an authorized administrator of Scholar Commons. For more information, please contact digres@mailbox.sc.edu. 


\title{
Long-lived spin coherence states in semiconductor heterostructures
}

\author{
Yuriy V. Pershin \\ Center for Quantum Device Technology, Department of Physics and Department of Electrical and Computer Engineering, \\ Clarkson University, Potsdam, New York 13699-5721, USA \\ and Department of Physics and Astronomy, Michigan State University, East Lansing, Michigan 48824-2320, USA
}

(Received 16 July 2004; revised manuscript received 4 January 2005; published 20 April 2005)

\begin{abstract}
We study evolution of electron spin coherence having nonhomogeneous direction of spin polarization vector in semiconductor heterostructures. It is found that the electron spin relaxation time due to the D'yakonovPerel' relaxation mechanism essentially depends on the initial spin polarization distribution. This effect has its origin in the coherent spin precession of electrons diffusing in the same direction. We predict a long spin relaxation time of a novel structure: a spin coherence standing wave and discuss its experimental realization.
\end{abstract}

DOI: 10.1103/PhysRevB.71.155317

PACS number(s): 72.15.Lh, 72.25.Dc, 85.75.-d

There is growing interest in the emerging field of spintronics with the aim of controlling and manipulating electron spins in microelectronic devices. Major achievements were attained in the metal spintronics, exploiting the giant magnetoresistance and tunnelling magnetoresistance effects in ferromagnetic-metal-layer and metal-insulator-metal structures. ${ }^{1,2}$ A number of metal spintronic devices are already commercialized, for example, magnetic field sensors, ${ }^{3}$ hard disk recording heads, and magnetic random access memory. Significant experimental and theoretical progress in semiconductor spin structures has been reported recently. ${ }^{4-14}$ Current research in semiconductor spintronics is mainly focused on spin injection ${ }^{7}$ and spin control, ${ }^{8,9}$ including manipulations of spin relaxation time. Long spin relaxation times are desirable for spintronic device operation.

Of particular interest in semiconductor spintronics are effects of spin-orbit interaction. ${ }^{15-28}$ On the one hand, electrically controlled spin-orbit interaction can be used for spin coherence manipulation, as with one of the most prominent device proposals - the spin-field-effect transistor of Datta and Dass. ${ }^{25}$ On the other hand, spin-orbit interaction causes electron spin relaxation. The corresponding relaxation mechanism is called D'yakonov-Perel' relaxation mechanism, ${ }^{29,30}$ arising from bulk asymmetry of a crystal (as with zincblende semiconductors) and/or asymmetry of confining potential. The D'yakonov-Perel' relaxation mechanism is identified as the leading spin relaxation mechanism in many important situations. All previous studies of electron spin relaxation in two-dimensional semiconductor heterostructures at zero applied electric field have focused either on properties of spatially homogeneous or spatially inhomogeneous spin polarization but with the same direction of spin polarization vector.

In this paper we investigate how the initial distribution of direction of spin polarization affects the spin lifetimes for electrons in quantum wells. The system under investigation is electrons in 2DEG with specific distribution and orientation of electron spins at initial moment of time. Specifically, the spin dynamics of two initial spin configurations, namely a spin polarization strip and a structure-spin coherence standing wave, is studied. In the spin coherence standing wave, the initial direction of the spin polarization is a periodic function of coordinate. We show that such a structure is more robust against relaxation than the electron spin polarization having the same direction of the spin polarization vector. This interesting phenomenon could open a different approach to semiconductor spintronic device operation.

The dynamics of electron spin polarization in an infinite 2DEG is modelled using a Monte Carlo simulation program described in Ref. 16 and subsequently used by us for studies of spin relaxation in 2 DEG with an antidote lattice. ${ }^{23}$ Within the Monte Carlo simulation algorithm, the space motion of 2DEG electrons is considered in the framework of the semiclassical approximation. The electrons are treated as classical particles, except that their kinetic energies are determined in the effective-mass approximation, which accounts for the semiconductor energy bands. We assume that electrons move along linear trajectories interrupted by the bulk scattering events. Our modelling involves spin-independent bulk scattering processes, which could be caused, e.g., by phonon scatterings or impurities. For the sake of simplicity, the scattering due to such events is assumed to be elastic and isotropic, i.e., the magnitude of the electron velocity is conserved in the scattering, while the final direction of the velocity vector is randomly selected. The time scale of the bulk scattering events can then be fully characterized by a single rate parameter, ${ }^{16}$ the momentum relaxation time, $\tau_{p}$. It is connected to the mean free path by $L_{p}=|\vec{\nu}| \tau_{p}$. Here $|\vec{\nu}|$ is the mean electron velocity.

In our simulations the electron spin polarization is conveniently described by the spin polarization vector $\vec{S}=\operatorname{Tr}(\rho \vec{\sigma})$, where $\rho$ is the single-electron density matrix. ${ }^{31}$ We consider the spin Hamiltonian consisting solely of the Rashba spinorbit term ${ }^{32}$

$$
H_{R}=\alpha \hbar^{-1}\left(\sigma_{x} p_{y}-\sigma_{y} p_{x}\right),
$$

where $\alpha$ is the interaction constant, $\vec{\sigma}$ is the Pauli-matrix vector corresponding to the electron spin, and $\vec{p}$ is the momentum of the electron confined in a two-dimensional geometry. It is assumed that $H_{R}$ influences only the spin coordinate, while the reciprocal effect of the spin on electron space motion is ignored. From the point of view of the electron spin, the effect of the Rashba spin-orbit coupling can be regarded as an effective magnetic field. In the presence of a 
magnetic field, the electron spin feels a torque and precesses in the plane perpendicular to the magnetic field direction with angular frequency $\vec{\Omega}$. Momentum scattering reorients the direction of the precession axis, making the orientation of the effective magnetic field random and trajectory dependent, thus leading to an average spin relaxation (dephasing). The quantum mechanical evolution of $\vec{S}$ can be described by the classical equation of motion $d \vec{S} / d t=\vec{\Omega} \times \vec{S} .^{31}$

To make a long story short, at the initial moment of time $t=0$ the electron coordinates $\vec{r}(t=0)$ and direction of velocity are randomly generated, while the spin direction is selected as described below. The main loop of the Monte Carlo simulation algorithm involves the following steps: generation of a time interval $t_{i+1}-t_{i}$ between two consecutive scattering events, calculation of $\vec{r}\left(t=t_{i+1}\right)$ and $\vec{S}\left(t=t_{i+1}\right)$ (using the classical equations of motion), and random generation of a new direction of electron velocity due to the scattering at $t=t_{i+1}$. Consecutive applications of these operations allow finding the electron position and spin direction at any arbitrary moment of time. We calculate $\langle\vec{S}\rangle$ as a function of time and coordinate by averaging over an ensemble of $10^{7}$ electrons. The spin relaxation time is evaluated by fitting the time dependence of $\langle\vec{S}\rangle$ to an exponential decay.

Mention should be made about the validity of the semiclassical approximation to the electron space motion. The semiclassical approximation is justified if the typical distance between impurities is much longer than the de Broglie wavelength, and the de Broglie wavelength is much longer than the unit cell. This hierarchy of lengths is met, for example, in lightly doped semiconductors and high-mobility heterostructures. The second requirement on the de Broglie wavelength implies that variations of the spin-orbit interaction on the unit cell length scale are averaged and the spin-orbit parameter $\alpha$ is considered as a space-independent constant. Examples of using the semiclassical approximation in modelling of spin-related phenomena in semiconductors can be found, e.g., in Refs. 16, 18, 19, and 23.

Evolution of a spin polarization strip. We start our consideration from a simple example, which will help to understand the main idea of our approach. Let us consider evolution of a spin polarization strip. We assume that at the initial moment of time $t=0$ the spin polarization is $\vec{S}=S_{0} \hat{z}$ for $\left|x-x_{0}\right|<a$ and 0 otherwise ( $\hat{z}$ axis is perpendicular to the heterostructure). Initial spin polarization is homogeneous in $y$ direction. Figure 1 shows results of our simulations. Evolution of spin polarization components in the central region of the strip is similar to the evolution of the homogeneous spin polarization [see Fig. 1(a)], $S_{z}$ component decreases with time and $S_{x}=S_{y}=0$. However, behavior of $S_{x}$ component near the edges of the strip is unusual, it has two pronounced peaks with amplitude comparable to $z$ component of spin polarization. These peaks have the same amplitude but different polarity.

The amplitude of the spin polarization as a function of coordinate is shown in Fig. 1(b). Peaks of spin polarization amplitude in the edge regions reveal that relaxation in these regions occurs appreciably slowly than in the bulk region. To understand this phenomenon, consider evolution of homoge-
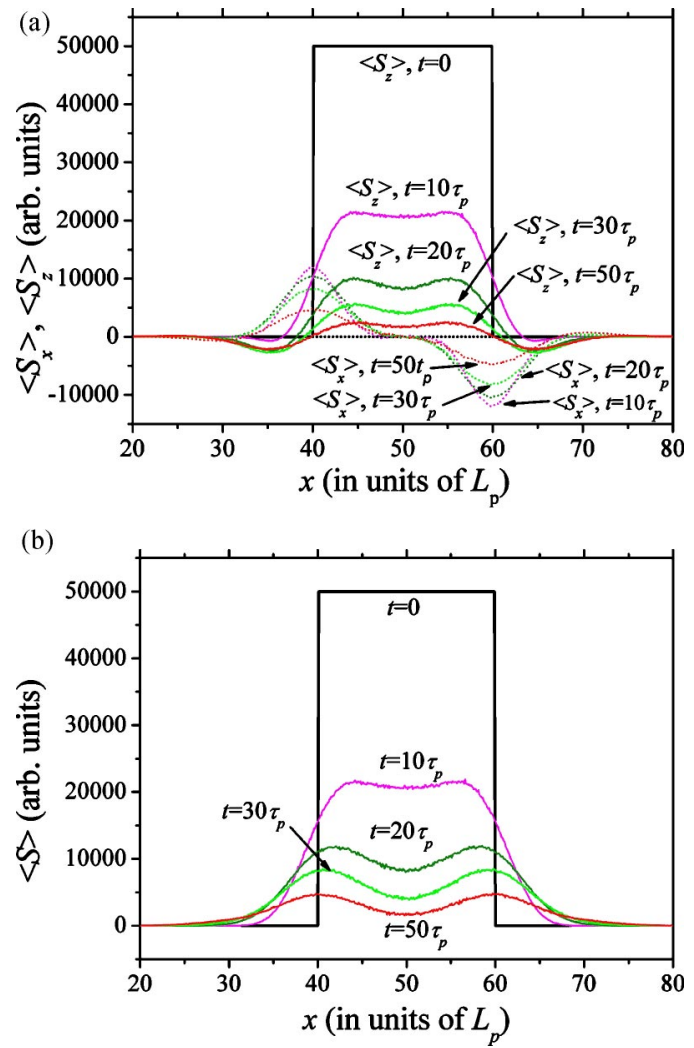

FIG. 1. (Color online) Evolution of the spin polarization strip, time dependence of spin polarization vector components (a) and amplitude (b).

neous spin polarization. The direction of electron spin precession between two consecutive scattering events is defined by the direction of electron motion. Since the system is homogeneous in $x$ and $y$ directions, the average $S_{x}$ and $S_{y}$ spin polarization components of electrons coming to an arbitrary selected space region are zero. When the symmetry of the system is broken, the transfer of spin polarization from $S_{z}$ to $S_{x}$ and $S_{y}$ may occur. Consider the electrons, for example, near the left edge of initial spin polarization profile. The polarized electrons diffuse out of the area of initial spin polarization, from right to left. Their spins precess coherently, and, since there are no spin-polarized electrons coming to this area from the opposite direction, $S_{x}$ component becomes uncompensated and conserved. This explains slower spin relaxation in the edge regions. The peaks of $S_{x}$ in Fig. 1(a) have a different polarity because of the opposite diffusion direction of spin-polarized electrons near the left and right edge. ${ }^{5}$ Using the Monte Carlo simulation algorithm we studied spin coherence evolution varying shape of initial spin polarization profile. It was found that the effect of spin polarization transfer to in-plane components is more prominent with decrease of space dimensions of areas with spin polarization gradients. Similar findings were observed by the authors of Ref. 15, who studied evolution of a pulse of spin polarization.

Spin coherence standing wave. Motivated by observation of longer spin relaxation time near the strip edges, we study evolution of a spin coherence standing wave, which is schematically shown in Fig. 2(a). Direction of spin polarization 


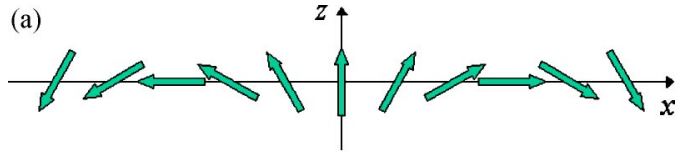

(b)

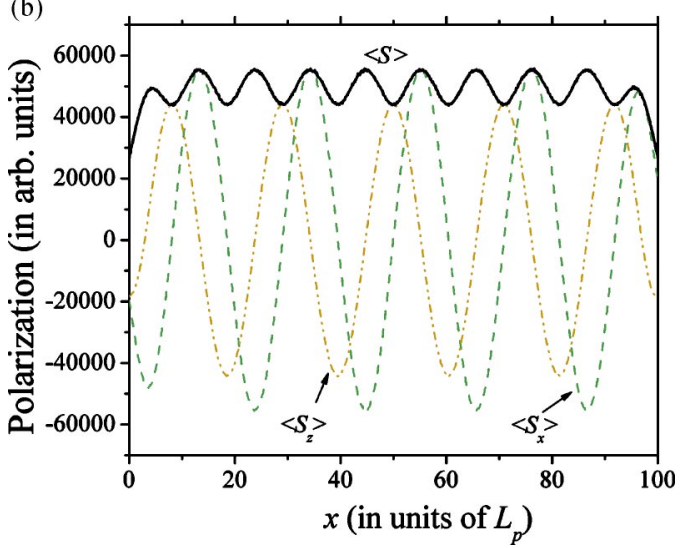

FIG. 2. (Color online) (a) Schematic of the spin coherence standing wave, direction of spin polarization vector is indicated by the arrows. (b) Total polarization and polarization components of the spin coherence standing wave at $t=5 \tau_{p}$, with $a=20.94 L_{p}$ and $\eta L_{p}=0.3$.

in the spin coherence standing wave is a periodic function of $x$ with the components $\left[-S_{0} \sin (2 \pi x / a), 0, S_{0} \cos (2 \pi x / a)\right]$, where $a$ is the period of the spin coherence standing wave and $S_{0}$ is its amplitude. Intuitively, the longest spin relaxation time will be, if after passing the distance $a$, the spin precession angle of an electron spin due to the Rashba spin-orbit interaction is equal to $2 \pi$. In this case the spin orientation of electrons moving along the $x$ direction will coincide with the initial direction of spin polarization vector and diffusion along the $x$ axis will not lead to relaxation. The electron spin relaxation will be associated only with the electron diffusion in the $y$ direction.

Figure 2(b) shows the distribution of the amplitude and components of spin coherence standing wave polarization at some time moment $t>0$. In our numerical simulations, the spin coherence standing wave was of a finite length, from $x=0$ to $x=100 L_{p}$, which explains the decrease of the spin coherence standing wave amplitude near the edges of this interval. However, we are mostly interested in evolution of spin coherence standing wave in the central region. It is found that in the central region the amplitude of spin coherence standing wave is a periodic function of $x$ with minimums corresponding to maximums of $S_{z}$ and with maximums corresponding to maximums of $S_{x}$. We attribute the transition from constant spin polarization amplitude at $t=0$ to a periodic one at subsequent time moments to the dependence of spin relaxation times on the initial direction of spin polarization vector. It is well known that spin relaxation time of in-plane spin polarization is two times longer then the spin relaxation time of the spin polarization perpendicular to the plane. $^{29}$

Spin relaxation time of the spin coherence standing wave as a function of its period is depicted in Fig. 3. This dependence has a maximum exactly at $a=2 \pi L_{p} / \eta$, where $\eta$ is the electron spin precession angle per mean free path. At the

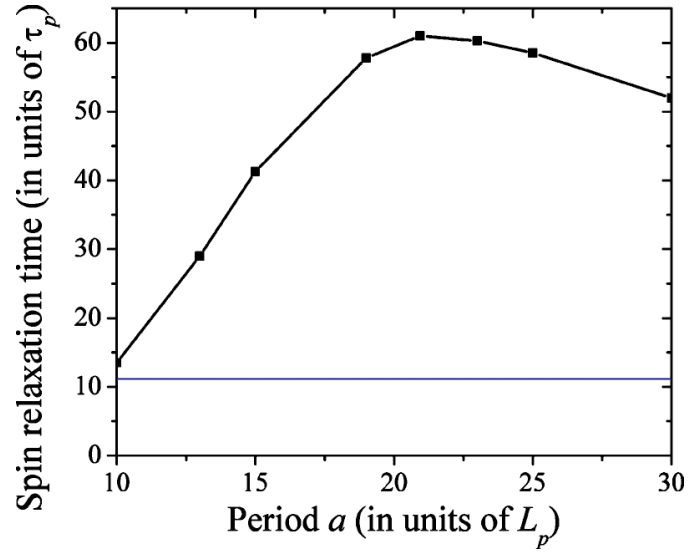

FIG. 3. Dependence of the electron spin relaxation time on the spin coherence standing wave period. The straight line shows the spin relaxation time of homogeneous spin polarization in the $z$ direction in the same system. This plot was obtained using the parameter value $\eta L_{p}=0.3$.

maximum, the relaxation time is 6 times as large for the spin coherence standing wave as for the homogeneous spin polarization in the $z$ direction. It is easy to understand why the spin relaxation time has a maximum at $a=2 \pi L_{p} / \eta$. Following the discussion above, if we consider an electron moving from $\vec{r}(t=0)=(x, y)$ to $\vec{r}\left(t=t_{1}\right)=(x+\delta x, y)$ then it is clear that the direction of $\vec{S}\left(t_{1}\right)$ coincides with the initial spin polarization of electrons at $(x+\delta x, y)$. Thus, at this particular value of $a$ the spin polarization dephasing due to the diffusion in the $x$ direction is completely eliminated, and the spin relaxation occurs because of the diffusion in the $y$ direction only.

We note that the dependence of spin relaxation time of spin coherence standing wave on its period could be useful for future spintronic applications. Potentially, this property allows transformation of space characteristics into the time domain. Moreover, spin coherence standing wave has another interesting property, namely, its phase. For instance, position of spin coherence standing wave minima could be used to encode the information. An applied electric field in $x$ direction induces sliding of the spin coherence standing wave allowing reading, writing, and manipulating the information.

From the experimental point of view, nonequlibrium spin polarization can be introduced into a semiconductor at the interface between semiconductor and ferromagnetic metal or magnetic semiconductor, or by using optical pumping techniques. ${ }^{12}$ Although both of these methods could be used to create a spin coherence standing wave, we concentrate our attention on the second method. Absorption of circularly polarized light in semiconductors generates spin-polarized electrons with spin polarization in the line of laser beam, while the direction of electron spin polarization (along or opposite to the beam) is determined by the light helicity. ${ }^{33}$ Two possible experimental setups are illustrated in Fig. 4. In the first case [Fig. 4(a)], a lattice of plain alternate right and left circularly polarized laser beams is used. After scattering on a light diffuser (such as, for example, opal diffusing glass), laser beams (applied in a pulse) polarize 2DEG electrons to a structure similar to the spin coherence standing wave. In the 

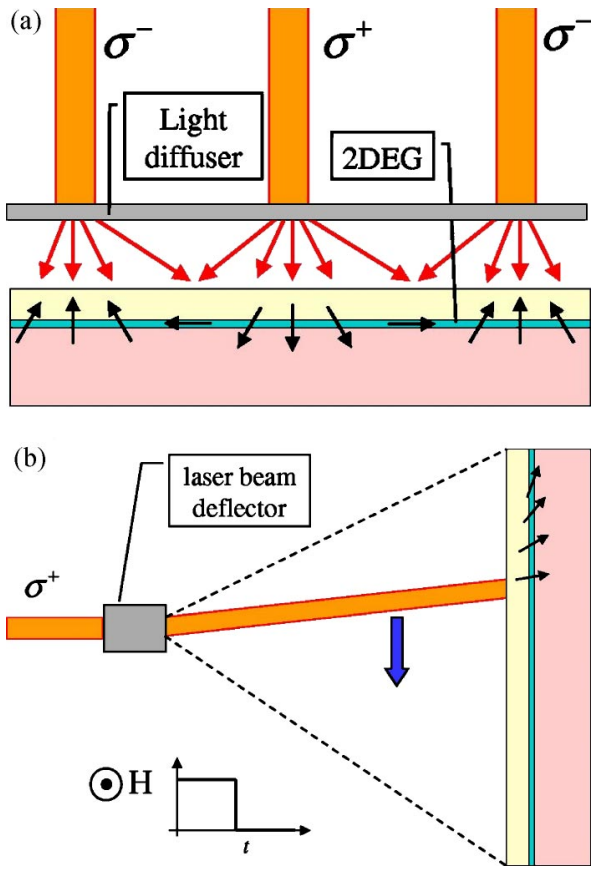

FIG. 4. (Color online) Experimental realization of the spin coherence standing wave via (a) a lattice of plain alternate right and left circularly polarized laser beams, and (b) deflected circularly polarized laser beam in a magnetic field. The black arrows in 2DEG region represent the electron spin polarization. The big arrow in (b) shows the direction of beam movement.

second experimental setup [Fig. 4(b)], circularly polarized laser beam travels from top to bottom of the sample with a constant velocity. Correspondingly, spin polarized electrons are created at different places at different moments of time. An external magnetic field is applied. The initial spin polarization direction of all electrons is nearly the same, however the electron spin precession angles due to the magnetic field are different for electrons excited at different moments of time. This allows obtaining the final spin configuration in the form of spin coherence standing wave. When the beam reaches the bottom of the sample, the magnetic field is switched off [Fig. 4(b)].

In conclusion, it was shown that the electron spin relaxation time in two-dimensional systems with inhomogeneous direction of electron spin polarization could be significantly longer as compared to the spin relaxation time in systems with homogeneous spin polarization. A structure, spin coherence standing wave characterized by periodicity of direction of spin polarization in one dimension was proposed and studied. Long spin relaxation time of this structure is explained by coherent spin precession of electrons diffusing in the same direction. Two distinctive features of the spin coherence standing wave, namely its long spin relaxation time and its phase, make it attractive for spintronic applications. Possible methods of spin coherence standing wave creation were discussed. It should be pointed out that the proposed structure allows reducing spin relaxation only due to D'yakonovPerel' relaxation mechanism. When D'yakonov-Perel' relaxation is reduced, other relaxation mechanism could be dominant. We can outline the following possible relaxation mechanisms: Elliot-Yafet, ${ }^{34,35}$ Bir-Aronov-Pikus, ${ }^{36}$ relaxation due to fluctuations of spin-orbit interaction, ${ }^{21}$ and relaxation by nuclear spins. ${ }^{37}$ Another possible source of spin dephasing is many body inhomogeneous broadening proposed by Wu. ${ }^{13}$ Investigation of joint action of these mechanisms is out of scope of this paper.

The author gratefully acknowledges helpful discussions with Professor V. Privman and Professor M. W. Wu. This research was supported by the National Security Agency and Advanced Research and Development Activity under Army Research Office Contract No. DAAD-19-02-1-0035, and by the National Science Foundation, Grant No. DMR-0121146. The author is also thankful to A.G. Mal'shukov for pointing out Ref. 38.
${ }^{1}$ G. Prinz, Phys. Today 48, 58 (1995).

${ }^{2}$ S. A. Wolf, D. D. Awschalom, R. A. Buhrman, J. M. Daughton, S. von Molnar, M. L. Roukes, A. Y. Chtchelkanova, and D. M. Treger, Science 294, 1488 (2001).

${ }^{3}$ S. Parkin, X. Jiang, C. Kaiser, A. Panchula, K. Roche, and M. Samant, Proc. IEEE 91, 661 (2003).

${ }^{4}$ S. Das Sarma, J. Fabian, X. Hu, and I. Zutic, Solid State Commun. 119, 207 (2001).

${ }^{5}$ D. D. Awschalom, M. E. Flatte, and N. Samarth, Sci. Am. 286, 66 (2002).

${ }^{6}$ H. Akinaga and H. Ohno, IEEE Trans. Nanotechnol. 1, 19 (2002).

${ }^{7}$ B. T. Jonker, Proc. IEEE 91, 727 (2003).

${ }^{8}$ J. B. Miller, D. M. Zumbuhl, C. M. Marcus, Y. B. Lyanda-Geller, D. Goldhaber-Gordon, K. Campman, and A. C. Gossard, Phys. Rev. Lett. 90, 076807 (2003).

${ }^{9}$ G. Salis, Y. Kato, K. Ensslin, D. C. Driscoll, A. C. Gossard, and D. D. Awschalom, Physica E (Amsterdam) 16, 99 (2003).
${ }^{10}$ J. M. Kikkawa, I. P. Smorchkova, N. Samarth, and D. D. Awschalom, Science 277, 1284 (1997).

${ }^{11}$ Y. Ohno, R. Terauchi, T. Adachi, F. Matsukura, and H. Ohno, Phys. Rev. Lett. 83, 4196 (1999).

${ }^{12}$ J. M. Kikkawa and D. D. Awschalom, Phys. Rev. Lett. 80, 4313 (1998); Nature (London) 397, 139 (1999).

${ }^{13}$ M. W. Wu, J. Supercond. 14, 245 (2001); M. Q. Weng and M. W. Wu, Phys. Rev. B 66, 235109 (2002); 68, 075312 (2003).

${ }^{14}$ Yu. V. Pershin and V. Privman, Phys. Rev. Lett. 90, 256602 (2003); Yu. V. Pershin, Phys. Rev. B 68, 233309 (2003).

${ }^{15}$ M. Q. Weng, M. W. Wu, and Q. W. Shi, Phys. Rev. B 69, 125310 (2004); M. W. Wu (private communication).

${ }^{16}$ A. A. Kiselev and K. W. Kim, Phys. Rev. B 61, 13115 (2000).

${ }^{17}$ A. M. Tyryshkin, S. A. Lyon, W. Jantsch, and F. Schaeffler, condmat/0304284 (unpublished).

${ }^{18}$ A. Bournel, P. Dollfus, E. Cassan, and P. Hesto, Appl. Phys. Lett. 77, 2346 (2000).

${ }^{19}$ S. Saikin, M. Shen, M.-C. Cheng, and V. Privman, J. Appl. Phys. 
94, 1769 (2003); S. Pramanik, S. Bandyopadhyay, and M. Cahay, Phys. Rev. B 68, 075313 (2003).

${ }^{20}$ R. G. Mani, J. H. Smet, K. von Klitzing, V. Narayanamurti, W. B. Johnson, and V. Umansky, Phys. Rev. B 69, 193304 (2004).

${ }^{21}$ E. Ya. Sherman, Phys. Rev. B 67, 161303(R) (2003); E. Ya. Sherman, Appl. Phys. Lett. 82, 209 (2003).

${ }^{22}$ Yu. V. Pershin, cond-mat/0310225 (unpublished).

${ }^{23}$ Yu. V. Pershin and V. Privman, Phys. Rev. B 69, 073310 (2004).

${ }^{24}$ M. Valín-Rodríguez, A. Puente, and L. Serra, Phys. Rev. B 69, 153308 (2004).

${ }^{25}$ S. Datta and B. Das, Appl. Phys. Lett. 56, 665 (1990).

${ }^{26}$ E. I. Rashba and Al. L. Efros, Phys. Rev. Lett. 91, 126405 (2003).

${ }^{27}$ F. X. Bronold, I. Martin, A. Saxena, and D. L. Smith, Phys. Rev. B 66, 233206 (2002).

${ }^{28}$ J. Schliemann, J. C. Egues, and D. Loss, Phys. Rev. Lett. 90, 146801 (2003).

${ }^{29}$ M. I. D'yakonov and V. I. Perel', Sov. Phys. Solid State 13, 3023
(1972).

${ }^{30}$ M. I. Dyakonov and V. Y. Kachorovskii, Sov. Phys. Semicond. 20, 110 (1986).

${ }^{31}$ K. Blum, Density Matrix Theory and Applications (Kluwer Academic, New York, 1996).

${ }^{32}$ E. I. Rashba, Fiz. Tverd. Tela (Leningrad) 2, 1224 (1960) [Sov. Phys. Solid State 2, 1109 (1960)]; Y. A. Bychkov and E. I. Rashba, J. Phys. C 17, 6039 (1984).

${ }^{33}$ Optical Orientation, edited by F. Meier and B. P. Zakharchenya (Elsevier Science, Amsterdam, 1984).

${ }^{34}$ P. G. Elliott, Phys. Rev. 96, 266 (1954).

${ }^{35}$ Y. Yafet, Solid State Phys. 14, 1 (1963).

${ }^{36}$ G. L. Bir, A. G. Aronov, and G. E. Pikus, Zh. Eksp. Teor. Fiz. 69, 1382 (1975) [Sov. Phys. JETP 42, 705 (1976)].

${ }^{37}$ Yu. V. Pershin and V. Privman, Nano Lett. 3, 695 (2003).

${ }^{38}$ A. G. Mal'shukov and K. A. Chao, Phys. Rev. B 61, R2413 (2000). 